\title{
Expression profiles and potential functions of circular RNAs in extracellular vesicles isolated from radioresistant glioma cells
}

\author{
MENGJIE ZHAO ${ }^{1 *}$, JINJIN XU², SHANLIANG ZHONG ${ }^{3}$, YUCHI LIU ${ }^{4}$, HONG XIAO $^{1}$, \\ LIANGYUAN GENG ${ }^{4}$ and HONGYI LIU ${ }^{4}$
}

${ }^{1}$ Department of Neuro-Psychiatric Institute, Affiliated Nanjing Brain Hospital, Nanjing Medical University, Nanjing 210029;

${ }^{2}$ Clinical Molecular Diagnostic Laboratory, The Second Affiliated Hospital of Nanjing Medical University, Nanjing 210003;

${ }^{3}$ Center of Clinical Laboratory Science, Jiangsu Cancer Hospital, Jiangsu Institute of Cancer Research,

The Affiliated Cancer Hospital of Nanjing Medical University, Nanjing 210009;

${ }^{4}$ Department of Neurosurgery, Affiliated Nanjing Brain Hospital, Nanjing Medical University,

Nanjing 210029, P.R. China

Received July 8, 2018; Accepted January 10, 2019

DOI: $10.3892 /$ or.2019.6972

\begin{abstract}
The molecular mechanisms of circular RNAs (circRNAs) in extracellular vesicles (EVs) associated with glioma radioresistance remain unknown. The aim of the present study was to assess the differential circRNA expression profiles between EVs isolated from U251 cells and EVs isolated from radioresistant U251 (RR-U251) cells. Identified circRNAs in EVs isolated from RR-U251 cells (RR-EVs) act as a U251 microRNA (miRNA) sponge. The circRNA expression was determined using RNA sequencing (RNA-seq) technique. A total of 1,235 circRNAs were detected. We identified 63 upregulated and 48 downregulated circRNAs in RR-EVs compared with those from U251 cells (Nor-EVs). The expression level of candidate circATP8B4 was confirmed using real-time quantitative PCR. It was significantly higher in RR-EVs than in Nor-EVs. Expression profile of RR-U251 and U251 miRNAs was conducted. miRanda and RNAhybrid
\end{abstract}

Correspondence to: Dr Liangyuan Geng or Professor Hongyi Liu, Department of Neurosurgery, Affiliated Nanjing Brain Hospital, Nanjing Medical University, Nanjing 210029, P.R. China

E-mail: cmugly@126.com

E-mail: hyliu18@126.com

*Contributed equally

Abbreviations: RR-U251, radioresistant U251 cells; circRNA, circular RNA; EVs, extracellular vesicles; Nor-EVs, EVs isolated from U251 cells; RR-EVs, EVs isolated from RR-U251 cells; RNA-seq, RNA sequencing; RT-qPCR, real-time quantitative PCR; miRNA, microRNA; GBM, glioblastoma; PBS, phosphate-buffered saline; GO, Gene Oncology; KEGG, Kyoto Encyclopedia of Genes and Genomes

Key words: circular RNA, glioma, radioresistance, extracellular vesicles, miRNA sponges, RNA sequencing softwares was used to predict the U251 downregulated miRNAs interacting with circATP8B4. CircATP8B4 from RR-EVs may be transferred to normal glioma U251 cells and act as an miR-766 sponge to promote cell radioresistance. In conclusion, using RNA-seq and bioinformatics, it was found that circATP8B4 in RR-EVs acts as a U251 miR-766 sponge, which may be involved in glioma radioresistance.

\section{Introduction}

Over the last decade, therapy for glioblastoma (GBM) has been centered on maximal safe surgical resection with adjuvant radiotherapy and chemotherapy. However, despite this breakthrough in combination therapy, the overall 5-year survival rate still remains less than $10 \%$ due to frequent tumor relapse $(1,2)$. Radiotherapy is a typical and aggressive approach for the treatment of many tumors, yet the inherent and acquired resistance of cancer cells is a major hurdle of radiotherapy (3). Growing evidence has demonstrated that radiation induces alterations in microRNA (miRNA) and gene profiles of many tumors (4). However, the underlying molecular mechanism of radioresistance is still not completely understood.

Extracellular vesicles (EVs, exosomes and microvesicles) are membrane-bound vessels that are actively secreted by mammalian cells. They contain a specific cargo of protein and nucleic acids (DNA, non-coding RNAs and mRNAs), which reflect their cell types of origin and may be used as biomarkers for the diagnosis of human diseases $(5,6)$. EVs have been recognized as important mediators of intercellular communication and they may play an important role in transporting radiation-induced RNAs and subsequently affect the proliferation and radioresistance of tumor cells (7).

Circular RNAs (circRNAs) have recently emerged as a comprehensive class of noncoding RNAs that may function as miRNA sponges and regulate gene expression in mammals. circRNAs are highly enriched in the brain and very stable within cells and body fluids $(8,9)$. Li et al reported that circRNAs are enriched and stable in liver cancer exosomes (10). Xu et al 
also found differential expression of circRNAs in EVs isolated from the serum of patients with endometrial cancer when compared with normal controls (11). A recent study demonstrated that exosomal circRNAs from arsenite-transformed cells were involved in the malignant transformation of human hepatic cells (12).

To the best of our knowledge, no studies to date have yet profiled the expression of circRNAs in EVs derived from radioresistant glioma. The function of circRNAs in EVs from radioresistant glioma is still unknown. Therefore, the aim of the present study was to investigate the differential circRNA expression in EVs isolated from radioresistant glioma cells and normal glioma cells in detail, and to identify circRNAs in EVs isolated from radioresistant glioma cells that may act as miRNA sponges that may be associated with glioma radioresistance using RNA sequencing and bioinformatics method.

\section{Materials and methods}

Cell culture. Human U-251MG cells (cat. no. KG050), obtained from Nanjing KeyGen Biotech Co., Ltd. (Nangjing, China; http://www.keygentec.com.cn/prd-search-U251.html), were cultured in Dulbecco's modified Eagle's medium (DMEM; Gibco-BRL; Therno Fisher Scientific, Inc., Waltham, MA, USA) containing $10 \%$ fetal bovine serum (FBS; Tianjin HaoYang Biological Manufacture Co., Ltd, Tianjing, China) and supplemented with penicillin-streptomycin. Cells were incubated at $37^{\circ} \mathrm{C}$ in $5 \% \mathrm{CO}_{2}$ and free of mycoplasma contamination after testing.

Establishment of radioresistant U251 cells (RR-U251). U251 cells were serially treated with $5 \mathrm{~Gy}$ of radiation using a ${ }^{60} \mathrm{Co}$ source (RuiDi Biotechnology, Nanjing, China) until 60 Gy of irradiation was reached, as previously described (13). Cell viability, cell migration and cell invasion experiments confirmed that radiosensitivity was decreased in RR-U251 cells and indicated the successful establishment of the RR-U251 cell line (13).

EV isolation. EVs were obtained from supernatants of U251 and RR-U251 cells cultured in DMEM with $10 \%$ exosome-free FBS. Cell culture media were sequentially centrifuged at $500 \mathrm{x} \mathrm{g}$ for $15 \mathrm{~min}, 16,500 \mathrm{x} \mathrm{g}$ for $30 \mathrm{~min}$ to remove floating cells and cellular debris followed by passing through a $0.22-\mu \mathrm{m}$ filter. The filtrates were further ultracentrifuged at $100,000 \mathrm{x} \mathrm{g}$ (Beckman Coulter Avanti J-30I; Beckman Coulter, Inc., Brea, CA, USA) for $2 \mathrm{~h}$ at $4^{\circ} \mathrm{C}$ and submitted to a second ultracentrifugation after washing in phosphate-buffered saline (PBS). The final EV pellets were further used immediately or resuspended in $100 \mu \mathrm{l} \mathrm{PBS}$ and stored at $-80^{\circ} \mathrm{C}$. EVs isolated from normal U251 and RR-U251 cells were abbreviated as Nor-EVs and RR-EVs, respectively.

Nanoparticle tracking analysis. Size determination of EVs was analyzed using ZetaView (Particle Matrix GmbH, Microtrac, Meerbusch, Germany).

Transmission electron microscopy. EVs $(10 \mu \mathrm{g})$ re-suspended in PBS were loaded onto Formvar/carbon coated grids (Ted
Pella Inc., Redding, CA, USA) fixed in $2.5 \%$ glutaraldehyde, contrasted in $2 \%$ uranyl acetate and visualized with FEI Tecnai G2 Spirit Bio TWIN ( FEI, Hillsboro, Oregon, USA).

RNA sequencing (RNA-seq). For RNA-seq, total RNA was extracted from U251, RR-U251 cells or EVs using the miRNeasy Micro kit (Qiagen GmbH, Hilden, Germany). The ribosomal RNA was removed from total RNA. After that, strand-specific RNA-seq library preparation was carried out using the VAHTS Total RNA-seq (H/M/R) Library Prep Kit for Illumina ${ }^{\circledR}$ (Vazyme Biotech Co., Ltd., Nanjing, China). In brief, RNA samples were fragmented into small pieces, and then used for first- and second-strand cDNA synthesis with random hexamer primers. In second-strand cDNA synthesis, dUTP mix (without dTTP) was used. After cDNA synthesis, end-repair was conducted, then a single 'A' base was added, and finally adaptors were ligated onto the cDNA products. Ligated cDNA products were purified and treated with uracil DNA glycosylase to remove the second-strand cDNA. For purified first-strand cDNA, PCR amplification was conducted followed by library quality analysis using Bioanalyzer 4200 (Agilent Technologies Inc., Santa Clara, CA, USA). Then the strand-specific RNA-seq libraries were sequenced on the HiSeq X10 system (Illumina, Inc., San Diego, CA, USA).

Differential expression analysis. HTSeq software version 0.6.1p2 (http://htseq.readthedocs.io/) was used to count reads mapped to genes. Differential expression level of circRNAs or miRNAs between the two groups was assessed by DEGseq algorithm in the statistical program $\mathrm{R}$ version 3.3.2 (http://www.r-project.org/). We identified differentially expressed circRNAs or miRNAs using a fold change $>1.5$. Those miRNAs with a zero read count were filtered.

Real-time quantitative polymerase chain reaction $(R T-q P C R)$. Total RNA was extracted and reverse transcribed using random primers by RevertAid First Strand cDNA Synthesis kit (Thermo Fisher Scientific, Inc., Waltham, MA, USA) according to the manufacturer's protocol. The relative circRNA expression levels were analyzed by FastStar Universal SYBR-Green Master (Roche Diagnostics, Indianapolis, IN, USA) on ABI 7300 (Thermo Fisher Scientific, Inc.). Divergent primer was designed for selected circRNA (circATP8B4: left primer CCACAGATGACT ATTTTCGCCA and right primer GGTCTCAACAGGTGG CCT). $\beta$-actin served as the internal reference to normalize the expression of circRNAs. The $2^{-\Delta \Delta C q}$ method (14) was used to calculate the expression level. Analysis was performed on three independent EV samples.

Prediction of circRNA-miRNA-mRNA network. The putative circRNA/miRNA interaction was predicted using miRanda software (August 2010 release, http://www.microrna. org/microrna/home.do) (15) and RNAhybrid software (http://bibiserv.techfak.uni-bielefeld.de/rnahybrid/) (16). miRanda was set according to the following parameters: gapopenpenalty was -9.0 , gapextendpenalty was -4.0 , score threshold was 140.0 , energy threshold was $1.0 \mathrm{kcal} / \mathrm{mol}$ and scaling parameter was 4.0. The following RNAhybrid setting parameters were used: miRNA and circRNA binding energy 
Table I. Top 20 differentially expressed circRNAs in EVs isolated from RR-U251 and U251 cells.

\begin{tabular}{|c|c|c|c|c|c|c|c|c|}
\hline circRNA & Chromosome & Start & End & Strand & $\begin{array}{l}\text { Exon } \\
\text { count }\end{array}$ & Gene name & Regulation & $\begin{array}{c}\text { Fold } \\
\text { change }\end{array}$ \\
\hline circATP8B4 & $\operatorname{chr} 15$ & 50038767 & 50107008 & - & 5 & АТР8B4 & Up & 2.972 \\
\hline $\operatorname{circCCDC} 134$ & $\operatorname{chr} 22$ & 41808874 & 41810291 & + & 3 & CCDC134 & $\mathrm{Up}$ & 2.972 \\
\hline circATP6V1H & chr8 & 53769617 & 53772167 & - & 2 & ATP6V1H & Up & 2.841 \\
\hline circMARCH6 & $\operatorname{chr} 5$ & 10415487 & 10417404 & + & 2 & MARCH6 & $\mathrm{Up}$ & 2.754 \\
\hline circLRRK1 & $\operatorname{chr} 15$ & 100983527 & 101015402 & + & 9 & LRRK1 & $\mathrm{Up}$ & 2.754 \\
\hline circANAPC1 & $\operatorname{chr} 2$ & 111808946 & 111825855 & - & 8 & ANAPC1 & Up & 2.637 \\
\hline circNSD2 & $\operatorname{chr} 4$ & 1918140 & 1918623 & + & 1 & NSD2 & Up & 2.637 \\
\hline circUBAP2 & $\operatorname{chr} 9$ & 33948373 & 33956146 & - & 3 & UBAP2 & $\mathrm{Up}$ & 2.475 \\
\hline circSEPT9 & $\operatorname{chr} 17$ & 77402058 & 77402703 & + & 1 & SEPT9 & Up & 2.475 \\
\hline circTBC1D1 & $\operatorname{chr} 4$ & 38089931 & 38103157 & + & 3 & TBC1D1 & $\mathrm{Up}$ & 2.475 \\
\hline circRNF216 & $\operatorname{chr} 7$ & 5641153 & 5652510 & - & 2 & RNF216 & Down & 2.972 \\
\hline circMTCL1 & chr18 & 8718423 & 8720496 & + & 2 & MTCL1 & Down & 2.754 \\
\hline circNR2C1 & $\operatorname{chr} 12$ & 95028386 & 95057878 & - & 7 & NR2C1 & Down & 2.754 \\
\hline circATAD2 & chr8 & 123333877 & 123336532 & - & 3 & ATAD2 & Down & 2.754 \\
\hline circCSPP1 & chr8 & 67105904 & 67116122 & + & 4 & CSPP1 & Down & 2.667 \\
\hline circUBQLN1 & $\operatorname{chr} 9$ & 83677726 & 83678599 & - & 2 & UBQLN1 & Down & 2.667 \\
\hline circ ZDHHC21 & $\operatorname{chr} 9$ & 14639895 & 14680162 & - & 6 & ZDHHC21 & Down & 2.625 \\
\hline circDLG1 & $\operatorname{chr} 3$ & 197090911 & 197119530 & - & 4 & DLG1 & Down & 2.625 \\
\hline circOSBPL10 & $\operatorname{chr} 3$ & 31876432 & 31879830 & - & 2 & OSBPL10 & Down & 2.584 \\
\hline $\operatorname{circSNX2}$ & $\operatorname{chr} 5$ & 122801868 & 122803613 & + & 3 & SNX2 & Down & 2.584 \\
\hline
\end{tabular}

RR-U251, radioresistant U251 cells; circRNA, circular RNA; EVs, extracellular vesicles; up, upregulated; down, downregulated.

$<-26 \mathrm{kcal} / \mathrm{mol}$; perfect miRNA seed complementarity with circRNA sequence, P-value $<0.05$. The candidate miRNA target gene was analyzed using miRanda and TargetScan software (http://www.targetscan.org/). In order to reduce the false positive rate, only the circRNA/miRNA interaction or miRNA target gene predicted by both two independent tools were taken into account.

EV labeling and coculture. EVs were labeled with the green fluorescent dye PKH67 (Sigma-Aldrich; Merck KGaA, Darmstadt, Germany) according to the manufacturer's protocol. Normal U251 cells were incubated with the PKH67-labeled RR-Evs in $37^{\circ} \mathrm{C}$ for $24 \mathrm{~h}$. Afterwards, the cocultured cells were washed twice and fixed with $4 \%$ paraformaldehyde for $30 \mathrm{~min}$ at room temperature. After counterstained with DAPI dihydrochloride (Beyotime Institute of Biotechnology, Haimen, China), fluorescence imaging was captured using confocal laser scanning microscopy (Carl Zeiss LSM710; Carl Zeiss, Oberkochen, Germany).

Cell viability assay. Cell viability was performed using MTT assay (Sigma-Aldrich; Merck KGaA). Following radiotherapy of $5 \mathrm{~Gy}$, normal U251 and the cocultured cells were seeded

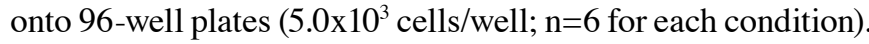
After 48 h, $20 \mu \mathrm{l}$ MTT was added to each well and incubated for $4 \mathrm{~h}$ prior to the solution of $150 \mu \mathrm{l}$ DMSO. Then the optical density (OD) in each individual well was recorded at $570 \mathrm{~nm}$ using a Multiskan Ascent microplate reader (Thermo Fisher
Scientific, Shanghai, China). Cell viability was calculated as follows: Cell viability $(\%)=\left(\mathrm{OD}_{\text {treatment }} / \mathrm{OD}_{\text {control }}\right) \times 100 \%$.

Statistical analysis. Statistical analyses were performed using GraphPad Prism 5 (GraphPad Software, Inc., La Jolla, CA, USA) and R software version 3.3.2. Student's t-test was used to determine significant differences between EVs from U251 and RR-U251 cells. A two-sided P-value $<0.05$ was considered to indicate a statistically significant result.

\section{Results}

Characterization of EVs released by U251 and RR-U251 cells. EVs released by the U251 and RR-U251 cells were isolated from the cell culture media (Materials and methods). They were characterized by the size and morphology using transmission electron microscopy and nanoparticle tracking analysis. The analyses confirmed the typical EV shape and size (Fig. 1A and B).

Identification of differential circRNA profiles and prediction of their characteristics. RNA-seq was performed to profile circRNA expression in a pair of Nor-EV and RR-EV samples. A total of 1,235 circRNAs were detected. Differentially expressed circRNAs were identified using fold change filtering (fold change $>1.5$ ). There were 63 circRNAs significantly upregulated in RR-EVs and 48 circRNAs were significantly downregulated in RR-EVs. The top 20 differentially expressed 
A

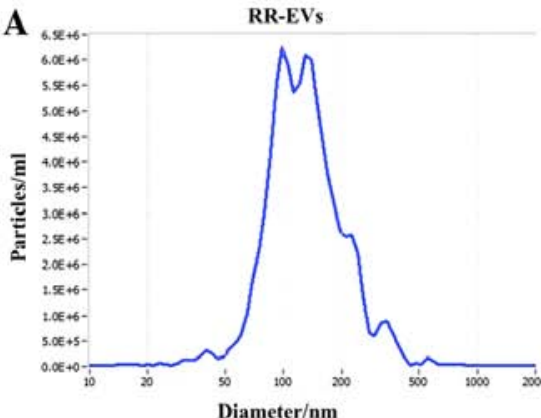

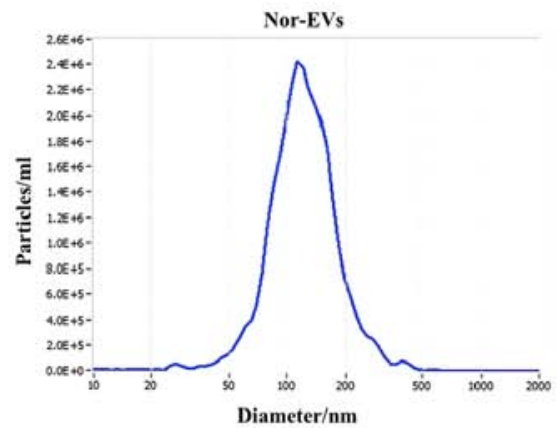

B

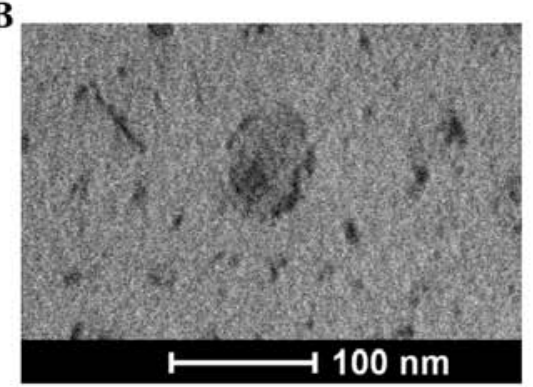

Figure 1. Characterization of EVs isolated from RR-U251 and U251 cells. (A) Determination of EV size distribution by nanoparticle tracking analysis. (B) Image of EVs from transmission electron microscopy. Scale bars, $100 \mathrm{~nm}$. RR-U251, radioresistant U251 cells; EVs, extracellular vesicles; Nor-EVs, EVs isolated from U251 cells; RR-EVs, EVs isolated from RR-U251 cells.

A

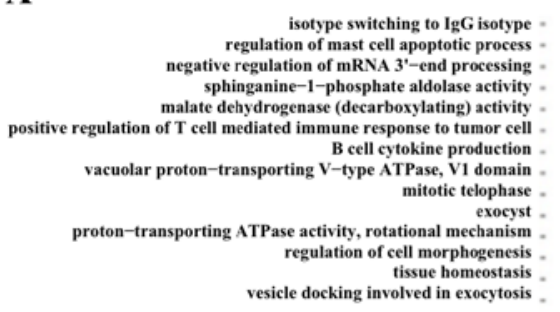

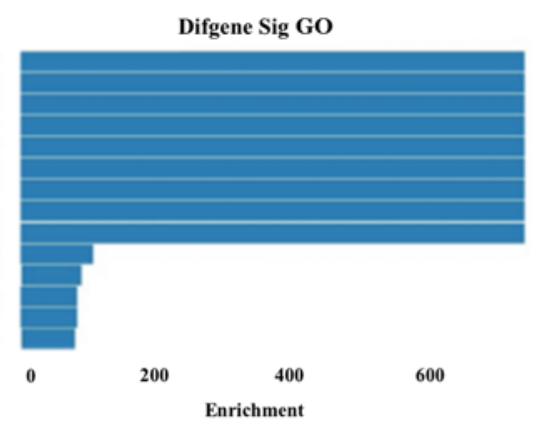

D

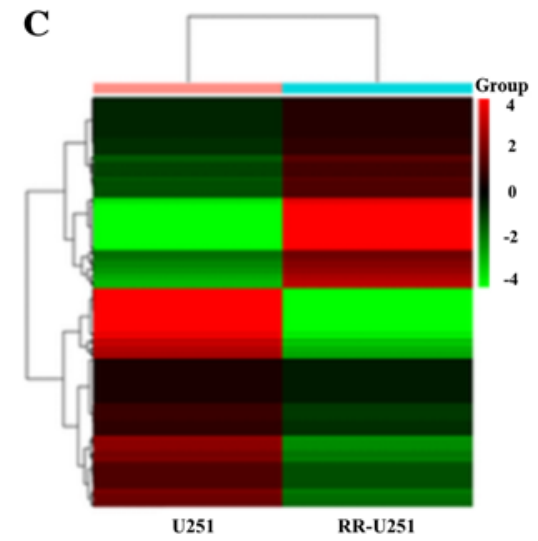

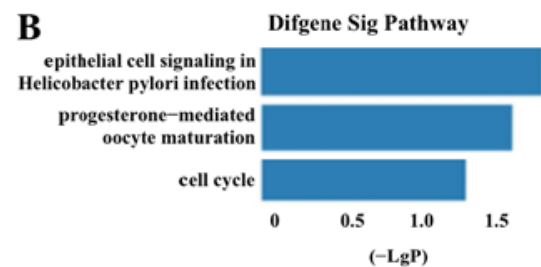

$(-\mathrm{LgP})$

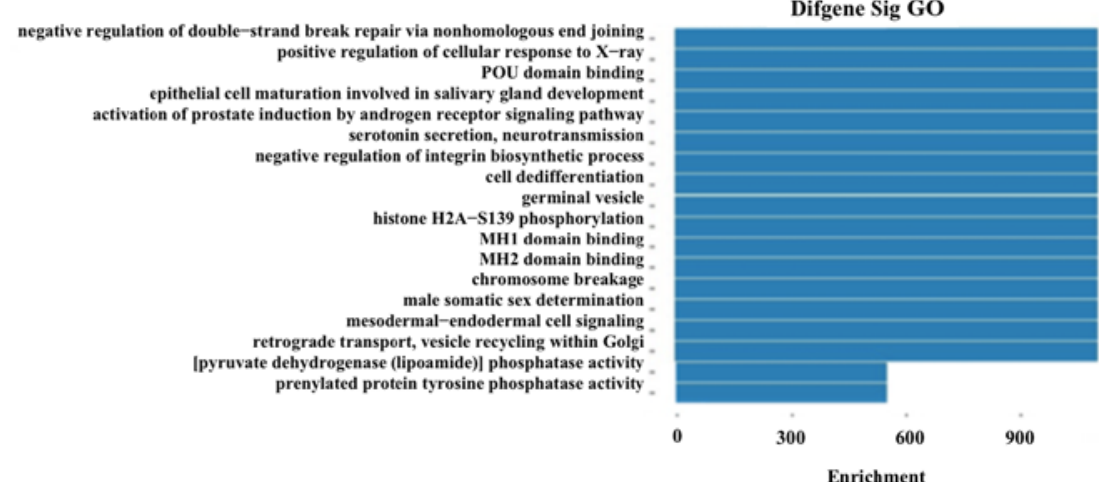

Figure 2. Characteristics of the differential circRNA and miRNA profiles. (A) GO and (B) KEGG pathway analysis of differential circRNAs in EVs isolated from RR-U251 cells and U251 cells. (C) Heatmap and (D) GO pathway of differential miRNA expression between U251 and RR-U251 cells. RR-U251, radioresistant U251 cells; circRNA, circular RNA; miRNA, microRNA; EVs, extracellular vesicles; GO, Gene Oncology; KEGG, Kyoto Encyclopedia of Genes and Genomes.

circRNAs are presented in Table I. We detected the functions of differentially expressed circRNAs in EV target genes using Gene Oncology (GO) and Kyoto Encyclopedia of Genes and Genomes (KEGG) pathway analysis. It showed that the target genes that were related to upregulated circRNAs participated in various biological processes, such as protein binding, regulation of mast cell apoptotic process and negative regulation of mRNA 3'-end processing (Fig. 2A). The upregulated circRNAs could also have an impact on several vital pathways, such as cell cycle pathway (Fig. 2B).

Identification of differential miRNA profiles and prediction of their characteristics. We analyzed miRNA expression in both U251 and RR-U251 cells based on RNA-seq. In total, 2,577
miRNAs were detected. Among these, 1,165 were present in RR-U251 cells; 1,170 were detected in U251 cells. We detected 171 upregulated and 184 downregulated miRNAs in the RR-U251 cells compared to the U251 cells. The top 20 differentially expressed miRNAs are presented in Table II. Heatmap was constructed to demonstrate the differential miRNA expression between U251 and RR-U251 cells (Fig. 2C). GO and KEGG pathway analysis was conducted. We found that the negative target mRNAs related to downregulated miRNAs were associated with various biological processes, such as protein binding, regulation of mast cell apoptotic process and positive regulation of cellular response to X-ray (Fig. 2D). The downregulated miRNAs could also have an impact on several vital pathways, such as the p53 signaling pathway. 
Table II. Top 20 differentially expressed miRNAs in U251 and RR-U251 cells.

\begin{tabular}{|c|c|c|c|c|}
\hline miRNA & $\log 2$ fold change & U251 & RR-U251 & Regulation \\
\hline hsa-miR-6787-3p & 3.887 & 0.937 & 13.867 & Up \\
\hline hsa-miR-665 & 3.646 & 0.937 & 11.734 & Up \\
\hline hsa-miR-6851-3p & 3.186 & 0.937 & 8.534 & Up \\
\hline hsa-miR-200b-5p & 2.771 & 0.937 & 6.400 & Up \\
\hline hsa-miR-4709-3p & 2.771 & 0.937 & 6.400 & Up \\
\hline hsa-miR-4717-3p & 2.771 & 0.937 & 6.400 & Up \\
\hline hsa-miR-3131 & 2.508 & 0.937 & 5.333 & Up \\
\hline hsa-miR-378g & 2.508 & 0.937 & 5.333 & Up \\
\hline hsa-miR-7110-3p & 2.186 & 0.937 & 4.267 & Up \\
\hline hsa-miR-3180-3p & 2.061 & 2.812 & 2.812 & Up \\
\hline hsa-miR-6739-3p & -2.984 & 8.437 & 1.067 & Down \\
\hline hsa-miR-548s & -2.984 & 8.437 & 1.067 & Down \\
\hline hsa-miR-6843-3p & -2.814 & 7.500 & 1.067 & Down \\
\hline hsa-miR-6854-5p & -2.814 & 7.500 & 1.067 & Down \\
\hline hsa-miR-5009-5p & -2.621 & 6.562 & 1.067 & Down \\
\hline hsa-miR-766-5p & -2.592 & 12.213 & 2.025 & Down \\
\hline hsa-miR-548u & -2.474 & 10.412 & 1.874 & Down \\
\hline hsa-miR-548a-3p & -2.474 & 5.206 & 0.937 & Down \\
\hline hsa-miR-3175 & -2.403 & 15.185 & 2.871 & Down \\
\hline hsa-miR-3130-5p & -2.403 & 7.593 & 1.436 & Down \\
\hline
\end{tabular}

RR-U251, radioresistant U251 cells; microRNA, miRNA; up, upregulated; down, downregulated.

A

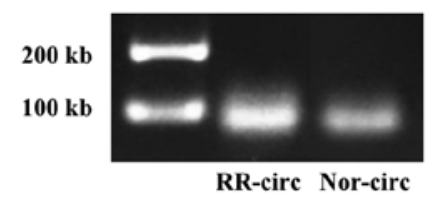

$\mathbf{C}$

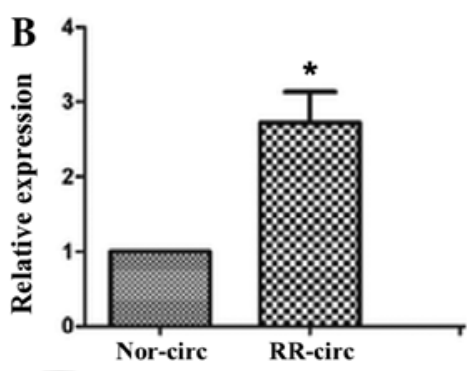

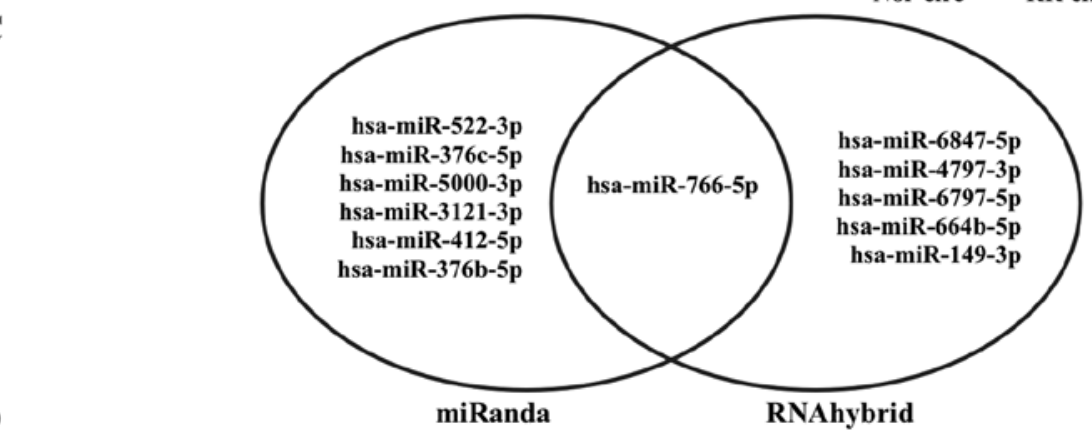

D

hsa-miR-766-5p vs chr15\#50038767-50107008-|ATP8B4

\section{miRanda}

Score: 151

Energy: $-20.25 \mathrm{kCa} 1 / \mathrm{Mo} 1$

Position: 246,265

miRNA 3' UUCUGGUCGUGGUUAAGGAGGA 5'
RNAhybrid

p-value: 0.030001

mfe: $-26.5 \mathrm{kCa} 1 / \mathrm{Mo} 1$

Position: 240, 266

miRNA 3' UUCUGGUCGU----GGUU----AAGGAGGA 5'

cirCRNA 5' ACAGCTAATTCCAGAAATTCCTCCTT 3'

Figure 3. RT-qPCR validation of the expression level of circATP8B4 in EVs and prediction of circRNA-miRNA interactions. (A) qPCR products of circATP8B4 were subjected to gel electrophoresis. (B) The average expression level of circATP8B4 was significantly higher in three independent EVs from radioresistant RR-U251 cells than those from U251 cells ( $\mathrm{P}=0.029)$. (C) The miRNAs interacting with circATP8B4 were predicted using miRanda and RNAhybrid softwares. (D) miR-766-5p was investigated. EVs, extracellular vesicles; circRNA, circular RNA; miRNA, microRNA; RR-circ, circATP8B4 in EVs isolated from RR-U251 cells; Nor-circ, circATP8B4 in EVs isolated from U251 cells. 
A

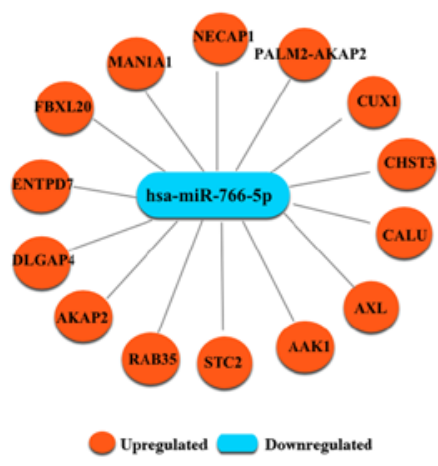

B

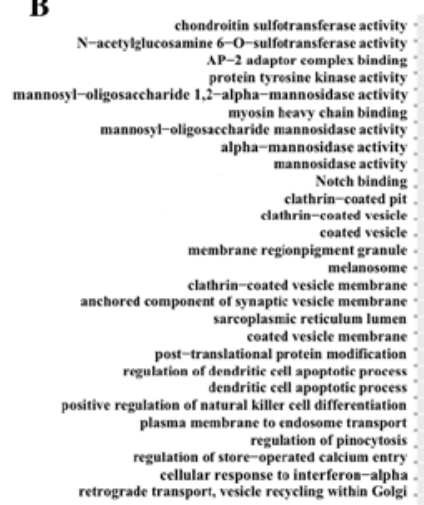

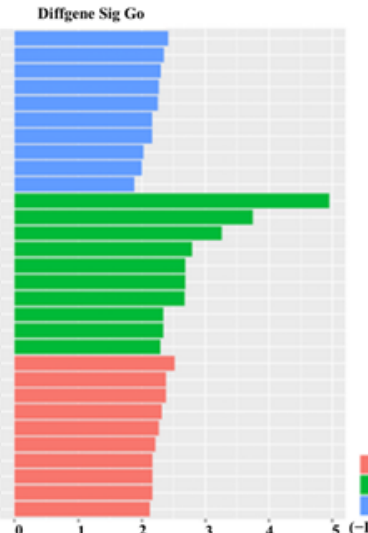

C

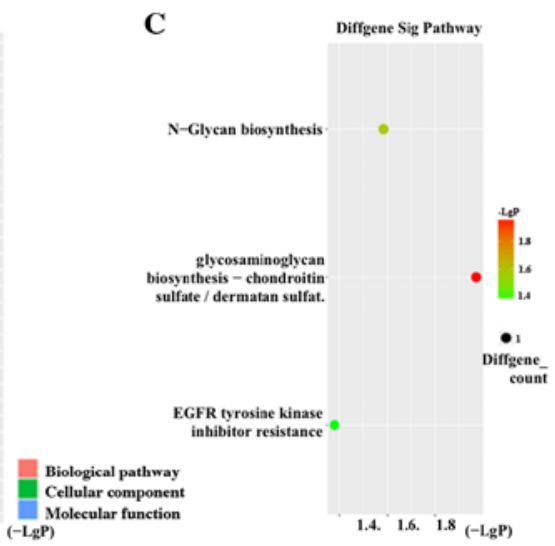

Figure 4. Characteristics of miR-766-5p negative target mRNAs. (A) Fourteen negative target mRNAs of miR-766-5p were predicted using miRanda and TargetScan software. (B) GO and (C) KEGG analysis was conducted. Gene Oncology; KEGG, Kyoto Encyclopedia of Genes and Genomes.

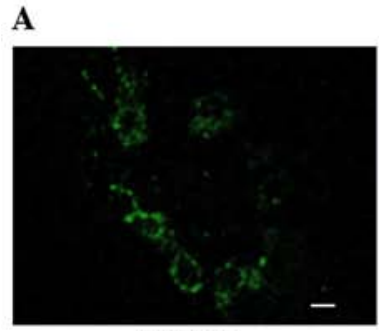

PKH67

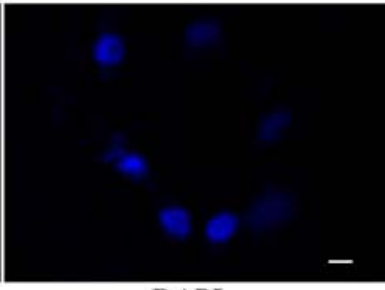

DAPI

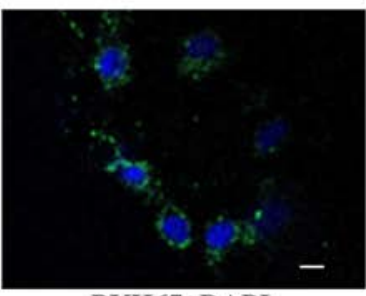

PKH67+DAPI

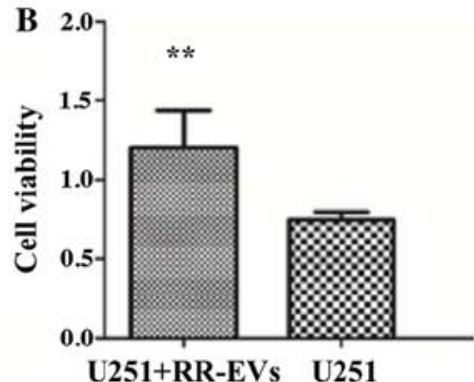

Figure 5. EV coculture and cell viability assay. (A) Normal U251 cells were incubated with the PKH67-labeled RR-EVs. After 24 h, RR-EVs were detected in the cocultured cells using confocal laser scanning microscopy. Scale bars, $20 \mu \mathrm{m}$. (B) The normal U251 and cocultured cells were treated with 5 Gy radiotherapy. MTT assay showed that cell viability of the cocultured cells was significantly higher than that of normal U251 cells (" $\mathrm{P}=0.001)$. RR-EVs, EVs isolated from RR-U251 cells.

Validation for the expression of circRNAs. To validate the RNA-seq results, RT-qPCR analysis was performed on one candidate circRNA in EVs with the top fold change. The candidate circATP8B4 is made up of five exons. The analysis was performed on three independent samples for RR-EVs and Nor-EVs, respectively. It showed that the average expression level of circATP8B4 was significantly higher in RR-EVs than those from Nor-EVs $(\mathrm{P}<0.05$; Fig. 3A and B).

Prediction of circRNA-miRNA-mRNA network. Literature has reported that circRNAs serve as miRNA sponges in the microenvironment and regulate gene expression. Therefore, we investigated those miRNAs with a fold change $>1.5$ in cells that could adsorb on circATP8B4. The sequence of circATP8B4 FASTA format was saved, and then we used miRanda and RNAhybrid softwares to predict the miRNAs interacting with circATP8B4. Only the circATP8B4-miRNA interactions predicted by both softwares were taken into account. The results showed that circATP8B4 may regulate miR-766-5p function as a microRNA sponge (Fig. 3C and D). Fourteen negative target mRNAs of miR-766-5p were predicted using miRanda and TargetScan software (Fig. 4A). There are two potential binding sites of miR-766-5p present in circATP8B4. GO analysis showed that the target genes were associated with many biological pathways, cellular components and molecular functions (Fig. 4B). They also could participate in many pathways such as EGFR tyrosine kinase inhibitor resistance according to KEGG analysis (Fig. 4C).

EV coculture and cell viability assay. In order to verify ceRNA mechanism of circATP8B4 preliminarily, normal U251 cells were incubated with the PKH67-labeled RR-EVs. After $24 \mathrm{~h}$, RR-EVs were detected in cocultured cells using confocal laser scanning microscopy (Fig. 5A). The normal U251 and cocultured cells were treated with 5-Gy radiotherapy. MTT assay demonstrated that cell viability of the cocultured cells was significantly higher than that of the normal U251 cells (Fig. 5B).

\section{Discussion}

Circular RNAs (circRNAs) exist widely in various organisms and are aberrantly expressed in epithelial tumors, such as bladder (17), breast cancer (18), basal cell carcinoma (19) and colorectal cancer (20) and in stromal tumors, such as glioma (21). Studies have reported that circRNAs are enriched in extracellular vesicles (EVs) isolated from liver cancer or serum of endometrial cancer patients $(10,11,20)$. Xu et al found that the number of upregulated circRNAs was more than the number of downregulated circRNAs in EVs from patients with endometrial cancer (11). In the present study, we profiled expression levels of circRNAs isolated from EVs in U251 and RR-U251 cells using RNA-seq, and a total of 111 differential 
circRNAs were identified. We detected 63 upregulated and 48 downregulated circRNAs in RR-EVs. Our data were similar to previous findings.

circRNAs are organization- and disease-specific, and can thus be potential biomarkers for disease diagnosis $(9,10,22-24)$. Zhu et al found that circBRAF was significantly downregulated in glioma patients with high pathological grade. The level of circBRAF was an independent biomarker for predicting survival in glioma patients (25). In the present study, to validate the RNA-seq findings, we performed RT-qPCR analysis for the most abundant candidate circRNA. The expression level of circATP8B4 was significantly higher in RR-EVs (EVs from radioresistant $\mathrm{U} 251$ cells) than this level in Nor-EVs (EVs from U251 cells). Thus, circATP8B4 from EVs could be a potential biomarker for glioma radioresistance.

Recent studies have found that circRNAs can regulate miRNA function as miRNA sponges and play a significant role in transcriptional control (26-29). Therefore, we predicted the downregulated miRNAs in U251 cells that could possibly interact with circATP8B4 using miRanda and RNAhybrid softwares, and candidate miR-766-5p was selected. GO and KEGG pathway analysis showed that the target genes that were related to downregulated miRNAs participated in various biological processes, such as cell apoptosis. Growing evidence indicates that miR-766 acts as a tumor promoter or suppressor in multiple cancer types (30-32). Afgar et al found that miR-766 can reactivate the expression of tumor-suppressor genes in colorectal cancer cell lines through DNMT3B gene inhibition (33). This indicates that circATP8B4 in RR-EVs might function as an miR-766 sponge to promote the proliferation and radioresistance of normal U251 cells.

Acquired radioresistance has been considered as one of the most important reasons for the treatment failure for GBM patients. Studies have shown that the tumor microenvironment is involved in the formation of glioma radioresistance $(3,34)$. In the tumor microenvironment, EVs are associated with cell-to-cell signaling transmission and may influence processes in glioma radioresistance. EVs derived from GBM cells were reported to promote temozolomide (TMZ) chemotherapy resistance of recipient cells via the transfer of cell-transforming proteins, mRNAs and miRNAs (35). However, the role of circRNAs in EVs associated with glioma radioresistant is still unclear. The present study showed that RR-EVs could be transferred to normal glioma U251 cells, and MTT assay showed those EVs are involved in the radioresistance of normal glioma cells. This implies that biomolecules such as ncRNAs or mRNAs in RR-EVs may play an important role in glioma radioresistance. Our preliminary experiments provide the possibility that circATP8B4 from RR-EVs may promote glioma radioresistance.

It should be noted that in the present study, we only identified one candidate upregulated circRNA in RR-EVs with the top fold change. Future research into the ceRNA mechanism of circATP8B4/miR-766/mRNA is still needed. The exact role of circATP8B4 in RR-EVs should be confirmed by overexpression and silencing experiments in vivo and in vitro. In addition, future clinical studies should be performed to verify the clinical significance of circATP8B4 in glioma radioresistance.

In conclusion, we identified 63 upregulated and 48 downregulated circRNAs in RR-EVs compared with those in
Nor-EVs. Gene Ontology and KEGG analyses were conducted to predict the characteristics of the circRNAs in EVs. We performed RT-qPCR analysis for candidate circATP8B4. CircATP8B4 from RR-EVs may be transferred to normal glioma U251 cells and circATP8B4 may act as an miR-766 sponge to promote cell radioresistance. Elucidation of the function and mechanism warrants further research.

\section{Acknowledgements}

Not applicable.

\section{Funding}

The present study was funded by the General Project of Nanjing Medical Science and Technology Development (no. YKK16145) and the Jiangsu Provincial Medical Innovation Team (no. CXTDA2017050).

\section{Availability of data and materials}

The datasets used and/or analyzed during the present study are available from the corresponding author on reasonable request.

\section{Authors' contributions}

LG and HL designed and conceived the study. MZ and JX performed the experiments; SZ, YL and HX assisted with the cell culture and EV isolation; JX and YL prepared the figures; SZ was responsible for the statistical analysis of the results. LG and MZ wrote the manuscript. LG, HL and HX reviewed and edited the manuscript and provided the funds. All authors read and approved the manuscript and agree to be accountable for all aspects of the research in ensuring that the accuracy or integrity of any part of the work are appropriately investigated and resolved.

\section{Ethics approval and consent}

Not applicable.

\section{Patient consent for publication}

Not applicable.

\section{Competing interests}

The authors declare that they have no competing interests.

\section{References}

1. Shah AH, Graham R, Bregy A, Thambuswamy $M$ and Komotar RJ: Recognizing and correcting failures in glioblastoma treatment. Cancer Invest 32: 299-302, 2014.

2. Quan JJ, Song JN and Qu JQ: PARP3 interacts with FoxM1 to confer glioblastoma cell radioresistance. Tumour Biol 36: 8617-8624, 2015

3. Han X, Xue X, Zhou H and Zhang G: A molecular view of the radioresistance of gliomas. Oncotarget 8: 100931-100941, 2017.

4. Li W, Guo F, Wang P, Hong S and Zhang C: miR-221/222 confers radioresistance in glioblastoma cells through activating Akt independent of PTEN status. Curr Mol Med 14: 185-195, 2014. 
5. Pant S, Hilton $\mathrm{H}$ and Burczynski ME: The multifaceted exosome: Biogenesis, role in normal and aberrant cellular function, and frontiers for pharmacological and biomarker opportunities. Biochem Pharmacol 83: 1484-1494, 2012.

6. Yang SJ, Wang DD, Li J, Xu HZ, Shen HY, Chen X, Zhou SY, Zhong SL, Zhao JH and Tang JH: Predictive role of GSTP1-containing exosomes in chemotherapy-resistant breast cancer. Gene 623: 5-14, 2017.

7. Tang Y, Cui Y, Li Z, Jiao Z, Zhang Y, He Y, Chen G, Zhou Q, Wang W, Zhou X, et al: Radiation-induced miR-208a increases the proliferation and radioresistance by targeting p21 in human lung cancer cells. J Exp Clin Cancer Res 12: 35-37, 2016.

8. Barbagallo D, Caponnetto A, Cirnigliaro M,Brex D, Barbagallo C, D'Angeli F, Morrone A, Caltabiano R, Barbagallo GM, Ragusa M, et al: CircSMARCA5 inhibits migration of glioblastoma multiforme cells by regulating a molecular axis involving splicing factors SRSF1/SRSF3/PTB. Int J Mol Sci 19: E480, 2018.

9. Li G, Yang H, Han K, Zhu D, Lun P and Zhao Y: A novel circular RNA, hsa_circ_0046701, promotes carcinogenesis by increasing the expression of miR-142-3p target ITGB8 in glioma. Biochem Biophys Res Commun 498: 254-261, 2018.

10. Li Y, Zheng Q, Bao C, Li S, Guo W, Zhao J, Chen D, Gu J, He X and Huang S: Circular RNA is enriched and stable in exosomes: A promising biomarker for cancer diagnosis. Cell Res 25: 981-984, 2015.

11. Xu H, Gong Z, Shen Y, Fang Y and Zhong S: Circular RNA expression in extracellular vesicles isolated from serum of patients with endometrial cancer. Epigenomics 10: 187-197, 2018.

12. Dai X, Chen C, Yang Q, Xue J, Chen X, Sun B, Luo F, Liu X, Xiao T, Xu H, et al: Exosomal circRNA_100284 from arsenite-transformed cells, via microRNA-217 regulation of EZH2, is involved in the malignant transformation of human hepatic cells by accelerating the cell cycle and promoting cell proliferation. Cell Death Dis 9: 454, 2018.

13. Du HQ, Wang Y, Jiang Y, Wang CH, Zhou T, Liu HY and Xiao H: Silencing of the TPM1 gene induces radioresistance of glioma U251 cells. Oncol Rep 33: 2807-2814, 2015.

14. Livak KJ and Schmittgen TD: Analysis of relative gene expression data using real-time quantitative PCR and the $2^{-\Delta \Delta C_{\mathrm{T}}}$ method. Methods 25: 402-408, 2001.

15. Enright AJ, John B, Gaul U, Tuschl T, Sander C and Marks DS: MicroRNA targets in Drosophila. Genome Biol 5: R1, 2003.

16. Krüger J and Rehmsmeier M: RNAhybrid: microRNA target prediction easy, fast and flexible. Nucleic Acids Res 34: W451-W454, 2006.

17. Zhong Z, Lv M and Chen J: Screening differential circular RNA expression profiles reveals the regulatory role of circTCF25-miR-103a-3p/miR-107-CDK6 pathway in bladder carcinoma. Sci Rep 6: 30919, 2016.

18. Nair AA, Niu N, Tang X, Thompson KJ, Wang L, Kocher JP Subramanian S and Kalari KR: Circular RNAs and their associations with breast cancer subtypes. Oncotarget 7: 80967-80979, 2016.

19. Sand M, Bechara FG, Sand D, Gambichler T, Hahn SA, Bromba M, Stockfleth E and Hessam S: Circular RNA expression in basal cell carcinoma. Epigenomics 8: 619-632, 2016.
20. Xie H, Ren X, Xin S, Lan X, Lu G, Lin Y, Yang S, Zeng Z, Liao W, Ding YQ, et al: Emerging roles of circRNA_001569 targeting miR-145 in the proliferation and invasion of colorectal cancer. Oncotarget 7: 26680-26691, 2016.

21. Yuan Y, Jiaoming L, Xiang W, Yanhui L, Shu J, Maling G and Qing M: Analyzing the interactions of mRNAs, miRNAs, lncRNAs and circRNAs to predict competing endogenous RNA networks in glioblastoma. J Neurooncol 137: 493-502, 2018.

22. Dou Y, Cha DJ, Franklin JL, Higginbotham JN, Jeppesen DK, Weaver AM, Prasad N, Levy S, Coffey RJ, Patton JG, et al: Circular RNAs are down-regulated in KRAS mutant colon cancer cells and can be transferred to exosomes. Sci Rep 6: 37982, 2016.

23. Zhuo F, Lin H, Chen Z, Huang Z and Hu J: The expression profile and clinical significance of circRNA0003906 in colorectal cancer. Onco Targets Ther 25: 5187-5193, 2017.

24. Zhang Y, Liang W, Zhang P, Chen J, Qian H, Zhang X and Xu W: Circular RNAs: Emerging cancer biomarkers and targets. J Exp Clin Cancer Res 36: 152, 2017.

25. Zhu J, Ye J, Zhang L, Xia L, Hu H, Jiang H, Wan Z, Sheng F, Ma Y, Li W, et al: Differential expression of circular RNAs in glioblastoma multiforme and its correlation with prognosis. Transl Oncol 10: 271-279, 2017.

26. Rong D, Sun H, Li Z, Liu S, Dong C, Fu K, Tang W and Cao H: An emerging function of circRNA-miRNAs-mRNA axis in human diseases. Oncotarget 8: 73271-73281, 2017.

27. Zheng Q, Bao C, Guo W, Li S, Chen J, Chen B, Luo Y, Lyu D, $\mathrm{Li} \mathrm{Y}$, Shi G, et al: Circular RNA profiling reveals an abundant circHIPK 3 that regulates cell growth by sponging multiple miRNAs. Nat Commun 7: 11215, 2016.

28. Hansen TB, Jensen TI, Clausen BH, Bramsen JB, Finsen B, Damgaard CK and Kjems J: Natural RNA circles function as efficient microRNA sponges. Nature 495: 384-388, 2013.

29. Nan A, Chen L, Zhang N, Liu Z, Yang T, Wang Z, Yang C and Jiang Y: A novel regulatory network among LncRpa, CircRarl, MiR-671 and apoptotic genes promotes lead-induced neuronal cell apoptosis. Arch Toxicol 91: 1671-1684, 2017.

30. Li YC, Li CF, Chen LB, Li DD, Yang L, Jin JP and Zhang B: MicroRNA-766 targeting regulation of SOX6 expression promoted cell proliferation of human colorectal cancer. Onco Targets Ther 8: 2981-2988, 2015.

31. Li X, Shi Y, Yin Z, Xue X and Zhou B: An eight-miRNA signature as a potential biomarker for predicting survival in lung adenocarcinoma. J Transl Med 12: 159, 2014.

32. Liang H, Li X, Wang L, Yu S, Xu Z, Gu Y, Pan Z, Li T, Hu M, Cui $\mathrm{H}$, et al: MicroRNAs contribute to promyelocyte apoptosis in $\mathrm{As}_{2} \mathrm{O}_{3}$-treated APL cells. Cell Physiol Biochem 32: 1818-1829, 2013.

33. Afgar A, Fard-Esfahani P, Mehrtash A, Azadmanesh K, Khodarahmi F, Ghadir M and Teimoori-Toolabi L: MiR-339 and especially miR-766 reactivate the expression of tumor suppressor genes in colorectal cancer cell lines through DNA methyltransferase 3B gene inhibition. Cancer Biol Ther 17: 1126-1138, 2016.

34. Combs SE, Schmid TE, Vaupel P and Multhoff G: Stress response leading to resistance in glioblastoma - The need for innovative radiotherapy (iRT) concepts. Cancers 8: E15, 2016.

35. Mondal A, Kumari Singh D, Panda S and Shiras A: Extracellular vesicles as modulators of tumor microenvironment and disease progression in glioma. Front Oncol 7: 144, 2017. 